\title{
ON A HILBERT SPACE OF ENTIRE FUNCTIONS
}

\author{
I.KH. MUSIN
}

\begin{abstract}
We consider the Hilbert space $F_{\varphi}^{2}$ of entire functions of $n$ variables constructed by means of a convex function $\varphi$ in $\mathbb{C}^{n}$ depending on the absolute value of the variable and growing at infinity faster than $a|z|$ for each $a>0$. We study the problem on describing the dual space in terms of the Laplace transform of the functionals. Under certain conditions for the weight function $\varphi$, we obtain the description of the Laplace transform of linear continuous functionals on $F_{\varphi}^{2}$. The proof of the main result is based on using new properties of Young-Fenchel transform and one result on the asymptotics of the multi-dimensional Laplace integral established by R.A. Bashmakov, K.P. Isaev, R.S. Yulmukhametov.
\end{abstract}

Keywords: Hilbert space, Laplace transform, entire functions, convex functions, YoungFenchel transform.

Mathematics Subject Classification: 32A15, 42B10, 46E10, 46F05, 42A38

\section{INTRODUCTION}

1.1. Problem. Let $H\left(\mathbb{C}^{n}\right)$ be the space of entire functions in $\mathbb{C}^{n}, d \mu_{n}$ be the Lebesgue measure in $\mathbb{C}^{n}$ and for $u=\left(u_{1}, \ldots, u_{n}\right) \in \mathbb{R}^{n}\left(\mathbb{C}^{n}\right)$ we define abs $u:=\left(\left|u_{1}\right|, \ldots,\left|u_{n}\right|\right)$.

We denote by $\mathcal{V}\left(\mathbb{R}^{n}\right)$ the set of all convex functions $g$ in $\mathbb{R}^{n}$ such that

1) $g\left(x_{1}, \ldots, x_{n}\right)=g\left(\left|x_{1}\right|, \ldots,\left|x_{n}\right|\right),\left(x_{1}, \ldots, x_{n}\right) \in \mathbb{R}^{n}$;

2) the restriction $g$ on $[0, \infty)^{n}$ is non-decaying in each variable;

3) $\lim _{x \rightarrow \infty} \frac{g(x)}{\|x\|}=+\infty ;\|x\|$ is the Euclidean norm of a point $\left.x \in \mathbb{R}^{n}\right)$.

To each function $\varphi \in \mathcal{V}\left(\mathbb{R}^{n}\right)$ we associate the Hilbert space

$$
F_{\varphi}^{2}=\left\{f \in H\left(\mathbb{C}^{n}\right):\|f\|_{\varphi}=\left(\int_{\mathbb{C}^{n}}|f(z)|^{2} e^{-2 \varphi(\text { abs } z)} d \mu_{n}(z)\right)^{\frac{1}{2}}<\infty\right\}
$$

with the scalar product

$$
(f, g)_{\varphi}=\int_{\mathbb{C}^{n}} f(z) \overline{g(z)} e^{-2 \varphi(\operatorname{abs} z)} d \mu_{n}(z), f, g \in F_{\varphi}^{2} .
$$

If $\varphi(x)=\frac{\|x\|^{2}}{2}$, then $F_{\varphi}^{2}$ is the Fock space.

It is obvious that for each function $\varphi \in \mathcal{V}\left(\mathbb{R}^{n}\right)$ and each $\lambda \in \mathbb{C}^{n}$, the function $f_{\lambda}(z)=e^{\langle\lambda, z\rangle}$ belongs to $F_{\varphi}^{2}$. This is why for each linear continuous functional $S$ on the space $F_{\varphi}^{2}$, the function

$$
\hat{S}(\lambda)=S\left(e^{\langle\lambda, z\rangle}\right), \quad \lambda \in \mathbb{C}^{n},
$$

is well defined in $\mathbb{C}^{n}$; this function is the Laplace transform of the functional $S$. It is easy to see that $\hat{S}$ is an entire function.

By $\left(F_{\varphi}^{2}\right)^{*}$ we denote the dual space for $F_{\varphi}^{2}$.

I.Kh. Musin, On a Hilbert space of entire functions.

(c) Musin I.KH.. 2017.

The work is financially supported by the Russian Foundation for Basic Researches (grant no. 15-01-01661) and the Program of the Presidium of RAS (project "Complex Analysis and Functional Equations".)

Submitted May 22, $201 \%$. 
The aim of the work is to find the conditions for $\varphi \in \mathcal{V}\left(\mathbb{R}^{n}\right)$, under which the space $\widehat{\left(F_{\varphi}^{2}\right)^{*}}$ of the Laplace transforms of the linear continuous functionals on $F_{\varphi}^{2}$ can be described as $F_{\varphi^{*}}^{2}$.

If $\varphi(x)=\frac{\|x\|^{2}}{2}$, then $\widehat{\left(F_{\varphi}^{2}\right)^{*}}=F_{\varphi}^{2}$. Indeed, in this case the problem on describing the space $\left(F_{\varphi}^{2}\right)^{*}$ in terms of the Laplace transform of the functionals is easily solved thanks to the classical representation: for each $f \in F_{\varphi}^{2}$,

$$
f(\lambda)=\pi^{-n} \int_{\mathbb{C}^{n}} f(z) e^{\langle\lambda, \bar{z}\rangle-\|z\|^{2}} d \mu_{n}(z), \quad \lambda \in \mathbb{C}^{n} .
$$

If the function $\varphi \in \mathcal{V}\left(\mathbb{R}^{n}\right)$ is radial, the mentioned problem was solved by V.V. Napalkov and S.V. Popenov [5], [6].

1.2. Notations and definitions. For $u=\left(u_{1}, \ldots, u_{n}\right), v=\left(v_{1}, \ldots, v_{n}\right) \in \mathbb{R}^{n}\left(\mathbb{C}^{n}\right)$ we let $\langle u, v\rangle:=u_{1} v_{1}+\cdots+u_{n} v_{n},\|u\|$ is the Euclidean norm of $u$.

Given $\alpha=\left(\alpha_{1}, \ldots, \alpha_{n}\right) \in \mathbb{Z}_{+}^{n}, z=\left(z_{1}, \ldots, z_{n}\right) \in \mathbb{C}^{n}$, by $|\alpha|:=\alpha_{1}+\ldots+\alpha_{n}$ we denote the length of the multi-index $\alpha, \tilde{\alpha}:=\left(\alpha_{1}+1, \ldots, \alpha_{n}+1\right)$, and we denote $z^{\alpha}:=z_{1}^{\alpha_{1}} \cdots z_{n}^{\alpha_{n}}$, $D_{z}^{\alpha}:=\frac{\partial^{|\alpha|}}{\partial z_{1}^{\alpha_{1} \ldots \partial z_{n}^{\alpha}}}$.

Given $\alpha=\left(\alpha_{1}, \ldots, \alpha_{n}\right) \in \mathbb{Z}_{+}^{n}, \varphi \in \mathcal{V}\left(\mathbb{R}^{n}\right)$, we define

$$
c_{\alpha}(\varphi):=\int_{\mathbb{C}^{n}}\left|z_{1}\right|^{2 \alpha_{1}} \cdots\left|z_{n}\right|^{2 \alpha_{n}} e^{-2 \varphi(\operatorname{abs} z)} d \mu_{n}(z) .
$$

For a function $u$ with a domain containing the set $(0, \infty)^{n}$, we define a function $u[e]$ in $\mathbb{R}^{n}$ by the rule:

$$
u[e](x)=u\left(e^{x_{1}}, \ldots, e^{x_{n}}\right), x=\left(x_{1}, \ldots, x_{n}\right) \in \mathbb{R}^{n} .
$$

By $\mathcal{B}\left(\mathbb{R}^{n}\right)$ we denote the set of all continuous functions $u: \mathbb{R}^{n} \rightarrow \mathbb{R}$ satisfying the condition

$$
\lim _{x \rightarrow \infty} \frac{u(x)}{\|x\|}=+\infty
$$

The Young-Fenchel transform of the function $u: \mathbb{R}^{n} \rightarrow[-\infty,+\infty]$ is the function $u^{*}: \mathbb{R}^{n} \rightarrow[-\infty,+\infty]$ defined by the formula

$$
u^{*}(x)=\sup _{y \in \mathbb{R}^{n}}(\langle x, y\rangle-u(y)), \quad x \in \mathbb{R}^{n} .
$$
then

If $E$ is a convex domain in $\mathbb{R}^{n}, h$ is a convex set in $E, \tilde{E}=\left\{y \in \mathbb{R}^{n}: h^{*}(y)<\infty\right\}, p>0$,

$$
D_{y}^{h}(p):=\left\{x \in E: h(x)+h^{*}(y)-\langle x, y\rangle \leqslant p\right\}, \quad y \in \tilde{E} .
$$

By $V(D)$ we denote the $n$-dimensional volume of a set $D \subset \mathbb{R}^{n}$.

\subsection{Main result.}

Theorem. Let $\varphi \in \mathcal{V}\left(\mathbb{R}^{n}\right)$ and for some $K>0$ and each $\alpha=\left(\alpha_{1}, \ldots, \alpha_{n}\right) \in \mathbb{N}^{n}$ the inequalities

$$
\frac{1}{K} \leqslant V\left(D_{\alpha}^{\varphi[e]}\left(\frac{1}{2}\right)\right) V\left(D_{\alpha}^{\varphi^{*}[e]}\left(\frac{1}{2}\right)\right) \prod_{j=1}^{n} \alpha_{j} \leqslant K
$$

hold. Then the mapping $\mathcal{L}: S \in\left(F_{\varphi}^{2}\right)^{*} \rightarrow \hat{S}$ makes an isomorphism between the spaces $\left(F_{\varphi}^{2}\right)^{*}$ and $F_{\varphi^{*}}^{2}$.

The proof of Theorem in Subsection 3.2 is based on new properties of Young-Fenchel transform, see Subsection 2.1, and one result on the asymptotics of the multi-dimensional Laplace integral in work [9], see Subsection 2.2. 


\section{Auxiliary DATA AND RESUlts}

2.1. On some properties of Young-Fenchel transform. It is easy to confirm that the following statement holds.

Proposition 1. Let $u \in \mathcal{B}\left(\mathbb{R}^{n}\right)$. Then $(u[e])^{*}(x)>-\infty$ as $x \in \mathbb{R}^{n},(u[e])^{*}(x)=+\infty$ as $x \notin[0, \infty)^{n}$ and $(u[e])^{*}(x)<+\infty$ as $x \in[0, \infty)^{n}$.

We note that the last statement of Proposition 1 is implied, for instance, by the fact that for each $M>0$ there exists a constant $A>0$ such that

$$
(u[e])^{*}(x) \leqslant \sum_{1 \leqslant j \leqslant n: x_{j} \neq 0}\left(x_{j} \ln \frac{x_{j}}{M}-x_{j}\right)+A, \quad x \in[0, \infty)^{n} .
$$

Proposition 2. Let $u \in \mathcal{B}\left(\mathbb{R}^{n}\right)$. Then

$$
\lim _{\substack{x \rightarrow \infty \\ x \in[0, \infty)^{n}}} \frac{(u[e])^{*}(x)}{\|x\|}=+\infty .
$$

Proof. For each $x \in[0, \infty)^{n}$ and $t \in \mathbb{R}^{n}$ we have

$$
(u[e])^{*}(x) \geqslant\langle x, t\rangle-(u[e])(t) .
$$

Employing this inequality, we obtain that for each $M>0$

$$
(u[e])^{*}(x) \geqslant M\|x\|-u[e]\left(\frac{M x}{\|x\|}\right), \quad x \in[0, \infty)^{n} \backslash\{0\} .
$$

This completes the proof.

The next three statements were proved in work [1], see there Lemma 6, Proposition 3, Proposition 4.

Proposition 3. Let $u \in \mathcal{B}\left(\mathbb{R}^{n}\right)$. Then

$$
\begin{aligned}
& (u[e])^{*}(x)+\left(u^{*}[e]\right)^{*}(x) \leqslant \sum_{\substack{1 \leqslant j \leqslant n: \\
x_{j} \neq 0}}\left(x_{j} \ln x_{j}-x_{j}\right), \quad x=\left(x_{1}, \ldots, x_{n}\right) \in[0, \infty)^{n} \backslash\{0\} ; \\
& (u[e])^{*}(0)+\left(u^{*}[e]\right)^{*}(0) \leqslant 0 .
\end{aligned}
$$

Proposition 4. Let $u \in \mathcal{B}\left(\mathbb{R}^{n}\right) \cap C^{2}\left(\mathbb{R}^{n}\right)$ be a convex function. Then

$$
(u[e])^{*}(x)+\left(u^{*}[e]\right)^{*}(x)=\sum_{j=1}^{n}\left(x_{j} \ln x_{j}-x_{j}\right), \quad x=\left(x_{1}, \ldots, x_{n}\right) \in(0, \infty)^{n} .
$$

Proposition 5. Let $u \in \mathcal{V}\left(\mathbb{R}^{n}\right) \cap C^{2}\left(\mathbb{R}^{n}\right)$ be a convex function. Then

$$
\begin{aligned}
& (u[e])^{*}(x)+\left(u^{*}[e]\right)^{*}(x)=\sum_{\substack{1 \leqslant j \leqslant n: \\
x_{j} \neq 0}}\left(x_{j} \ln x_{j}-x_{j}\right), \quad x=\left(x_{1}, \ldots, x_{n}\right) \in[0, \infty)^{n} \backslash\{0\} ; \\
& (u[e])^{*}(0)+\left(u^{*}[e]\right)^{*}(0)=0 .
\end{aligned}
$$

Propositions 4 and 5 can be strengthen by employing the results by D. Azagra [2], [3]. He proved the following theorem.

Theorem A. Let $U \subseteq \mathbb{R}^{n}$ be an open convex set. For each convex function $f: U \rightarrow \mathbb{R}$ and each $\varepsilon>0$ there exists a real analytic convex function $g: U \rightarrow \mathbb{R}$ such that

$$
f(x)-\varepsilon \leqslant g(x) \leqslant f(x), \quad x \in U .
$$

Thus, the following corollary hold [3]. 
Corollary A. Let $U \subseteq \mathbb{R}^{n}$ be an open convex set. For each convex function $f: U \rightarrow \mathbb{R}$ and each $\varepsilon>0$ there exists an infinitely differentiable convex function $g: U \rightarrow \mathbb{R}$ such that

$$
f(x)-\varepsilon \leqslant g(x) \leqslant f(x), \quad x \in U .
$$

Employing Proposition 4 and Corollary A, we easily confirm the following statement.

Proposition 6. Let $u \in \mathcal{B}\left(\mathbb{R}^{n}\right)$ be a convex function. Then

$$
(u[e])^{*}(x)+\left(u^{*}[e]\right)^{*}(x)=\sum_{j=1}^{n}\left(x_{j} \ln x_{j}-x_{j}\right), \quad x=\left(x_{1}, \ldots, x_{n}\right) \in(0, \infty)^{n} .
$$

Moreover, the following proposition is true.

Proposition 7. Let $u \in \mathcal{V}\left(\mathbb{R}^{n}\right)$ be a convex function. Then

$$
\begin{aligned}
& (u[e])^{*}(x)+\left(u^{*}[e]\right)^{*}(x)=\sum_{\substack{1 \leqslant j \leqslant n: \\
x_{j} \neq 0}}\left(x_{j} \ln x_{j}-x_{j}\right), \quad x=\left(x_{1}, \ldots, x_{n}\right) \in[0, \infty)^{n} \backslash\{0\} ; \\
& (u[e])^{*}(0)+\left(u^{*}[e]\right)^{*}(0)=0 .
\end{aligned}
$$

Proof. According Proposition 6, our statement is true for the points $x \in(0, \infty)^{n}$. Assume that $x=\left(x_{1}, \ldots, x_{n}\right)$ belongs to the boundary of $[0, \infty)^{n}$ and $x \neq 0$. For the sake of simplicity we consider the case when the first $k(1 \leqslant k \leqslant n-1)$ coordinates of $x$ are positive and all other are equal to zero. For each $\xi=\left(\xi_{1}, \ldots, \xi_{n}\right), \mu=\left(\mu_{1}, \ldots, \mu_{n}\right) \in \mathbb{R}^{n}$ we have

$$
(u[e])^{*}(x)+\left(u^{*}[e]\right)^{*}(x) \geqslant \sum_{j=1}^{k} x_{j}\left(\xi_{j}+\mu_{j}\right)-\left(u\left(e^{\xi_{1}}, \ldots, e^{\xi_{n}}\right)+u^{*}\left(e^{\mu_{1}}, \ldots, e^{\mu_{n}}\right)\right) .
$$

By this inequality we obtain that

$$
\begin{aligned}
(u[e])^{*}(x)+\left(u^{*}[e]\right)^{*}(x) & \geqslant \sum_{j=1}^{k} x_{j}\left(\xi_{j}+\mu_{j}\right) \\
& -\left(u\left(e^{\xi_{1}}, \ldots, e^{\xi_{k}}, 0, \ldots, 0\right)+u^{*}\left(e^{\mu_{1}}, \ldots, e^{\mu_{k}}, 0, \ldots, 0\right)\right) .
\end{aligned}
$$

We define a function $u_{k}$ on $\mathbb{R}^{k}$ by the rule: $\left(\lambda_{1}, \ldots, \lambda_{k}\right) \in \mathbb{R}^{k} \rightarrow u\left(\lambda_{1}, \ldots, \lambda_{k}, 0, \ldots, 0\right)$. We observe that for each $t=\left(t_{1}, \ldots, t_{k}\right) \in \mathbb{R}^{k}, \breve{t}=\left(t_{1}, \ldots, t_{k}, 0, \ldots, 0\right) \in \mathbb{R}^{n}$ we have

$$
\begin{aligned}
u^{*}(\breve{t}) & =\sup _{v \in \mathbb{R}^{n}}(\langle\breve{t}, v\rangle-u(v)) \\
& \leqslant \sup _{v_{1}, \ldots, v_{k} \in \mathbb{R}}\left(\sum_{j=1}^{k} t_{j} v_{j}-u\left(v_{1}, \ldots, v_{k}, 0, \ldots, 0\right)\right)=\sup _{v \in \mathbb{R}^{k}}\left(\langle t, v\rangle-u_{k}(v)\right)=u_{k}^{*}(t) .
\end{aligned}
$$

Employing this and the above inequality, for $\tilde{x}=\left(x_{1}, \ldots, x_{k}\right) \in \mathbb{R}^{k}$ and each $\tilde{\xi}=$ $\left(\xi_{1}, \ldots, \xi_{k}\right), \tilde{\mu}=\left(\mu_{1}, \ldots, \mu_{k}\right) \in \mathbb{R}^{k}$ we have

$$
(u[e])^{*}(x)+\left(u^{*}[e]\right)^{*}(x) \geqslant\langle\tilde{x}, \tilde{\xi}\rangle-u_{k}[e](\tilde{\xi})+\langle\tilde{x}, \tilde{\mu}\rangle-u_{k}^{*}[e](\tilde{\mu}) .
$$

Therefore,

$$
(u[e])^{*}(x)+\left(u^{*}[e]\right)^{*}(x) \geqslant\left(u_{k}[e]\right)^{*}(\tilde{x})+\left(u_{k}^{*}[e]\right)^{*}(\tilde{x}) .
$$

Since by the Proposition 6,

$$
\left(u_{k}[e]\right)^{*}(\tilde{x})+\left(u_{k}^{*}[e]\right)^{*}(\tilde{x})=\sum_{j=1}^{k}\left(x_{j} \ln x_{j}-x_{j}\right)
$$


then $(u[e])^{*}(x)+\left(u^{*}[e]\right)^{*}(x) \geqslant \sum_{j=1}^{k}\left(x_{j} \ln x_{j}-x_{j}\right)$. By Proposition 3 this implies the first statement of the proposition.

If $x=0$, then

$$
\begin{aligned}
(u[e])^{*}(0) & =-\inf _{\xi \in \mathbb{R}^{n}} u[e](\xi)=-u(0), \\
\left(u^{*}[e]\right)^{*}(0) & =-\inf _{\xi \in \mathbb{R}^{n}} u^{*}[e](\xi)=-u^{*}(0)=\inf _{\xi \in \mathbb{R}^{n}} u(\xi)=u(0) .
\end{aligned}
$$

Therefore, $(u[e])^{*}(0)+\left(u^{*}[e]\right)^{*}(0)=0$.

2.2. Asymptotics of multi-dimensional Laplace integral. In work [9] there was established the following theorem.

Theorem B. Let $E$ be a convex domain in $\mathbb{R}^{n}$, $h$ be a convex function in $E, \tilde{E}=\left\{y \in \mathbb{R}^{n}\right.$ : $\left.h^{*}(y)<\infty\right\}$ and the interior of $\tilde{E}$ is non-empty. Let

$$
\begin{aligned}
& D^{h}=\left\{(x, y) \in \mathbb{R}^{n} \times \mathbb{R}^{n}: h(x)+h^{*}(y)-\langle x, y\rangle \leqslant 1\right\}, \\
& D_{y}^{h}=\left\{x \in \mathbb{R}^{n}:(x, y) \in D\right\}, y \in \mathbb{R}^{n} .
\end{aligned}
$$

Then

$$
e^{-1} V\left(D_{y}^{h}\right) e^{h^{*}(y)} \leqslant \int_{\mathbb{R}^{n}} e^{\langle x, y\rangle-h(x)} d x \leqslant(1+n !) V\left(D_{y}^{h}\right) e^{h^{*}(y)}, \quad y \in \tilde{E} .
$$

Here we assume that $h(x)=+\infty$ as $x \notin E$.

\section{DESCRIPTION OF DUAL SPACE}

3.1. Auxiliary lemmata. In the proof of Theorem the following four lemmata will be useful.

Lemma 1. Let $\varphi \in \mathcal{V}\left(\mathbb{R}^{n}\right)$. Then the system $\{\exp \langle\lambda, z\rangle\}_{\lambda \in \mathbb{C}^{n}}$ is complete in $F_{\varphi}^{2}$.

Proof. Let $S$ be a linear continuous functional on the space $F_{\varphi}^{2}$ such that $S\left(e^{\langle\lambda, z\rangle}\right)=0$ for each $\lambda \in \mathbb{C}^{n}$. Since for each multi-index $\alpha \in \mathbb{Z}_{+}^{n}$ we have $\left(D_{\lambda}^{\alpha} \hat{S}\right)(\lambda)=S\left(z^{\alpha} e^{\langle z, \lambda\rangle}\right)$, this identity implies that $S\left(z^{\alpha}\right)=0$. Since the function $\varphi\left(\left|z_{1}\right|, \ldots,\left|z_{n}\right|\right)$ is convex in $\mathbb{C}^{n}$, it follows from the result by B.A. Taylor on the weight approximation of entire functions by polynomials 4 , Thm. 2] that the polynomials are dense in $F_{\varphi}^{2}$. Hence, $S$ is the zero functional. By the known corollary of Khan-Banach theorem we obtain that the system $\{\exp \langle\lambda, z\rangle\}_{\lambda \in \mathbb{C}^{n}}$ is complete in $F_{\varphi}^{2}$.

We note that the system $\left\{z^{\alpha}\right\}_{|\alpha| \geqslant 0}$ is orthogonal in $F_{\varphi}^{2}$. Moreover, it is dense in $F_{\varphi}^{2}$. Therefore, the system $\left\{z^{\alpha}\right\}_{|\alpha| \geqslant 0}$ is a basis in $F_{\varphi}^{2}$.

Lemma 2. Let $\varphi \in \mathcal{V}\left(\mathbb{R}^{n}\right)$. Then

$$
c_{\alpha}(\varphi) \geqslant \frac{\pi^{n}}{\tilde{\alpha}_{1} \cdots \tilde{\alpha}_{n}} e^{2(\varphi[e])^{*}(\tilde{\alpha})}, \quad \alpha \in \mathbb{Z}_{+}^{n} .
$$

In particular, for each $M>0$ there exists a constant $C_{M}>0$ such that $c_{\alpha}(\varphi) \geqslant C_{M} M^{|\alpha|}$ for each $\alpha \in \mathbb{Z}_{+}^{n}$ 
Proof. For each $\alpha \in \mathbb{Z}_{+}^{n}$ and each positive numbers $R_{1}, \ldots, R_{n}$ we have

$$
\begin{aligned}
c_{\alpha}(\varphi) & =(2 \pi)^{n} \int_{0}^{\infty} \cdots \int_{0}^{\infty} r_{1}^{2 \alpha_{1}+1} \cdots r_{n}^{2 \alpha_{n}+1} e^{-2 \varphi\left(r_{1}, \cdots, r_{n}\right)} d r_{1} \cdots d r_{n} \\
& \geqslant(2 \pi)^{n} \int_{0}^{R_{1}} \cdots \int_{0}^{R_{n}} r_{1}^{2 \alpha_{1}+1} \cdots r_{n}^{2 \alpha_{n}+1} e^{-2 \varphi\left(R_{1}, \cdots, R_{n}\right)} d r_{1} \cdots d r_{n} \\
& =(2 \pi)^{n} \frac{R_{1}^{2 \alpha_{1}+2}}{2 \alpha_{1}+2} \cdots \frac{R_{n}^{2 \alpha_{n}+2}}{2 \alpha_{n}+2} e^{-2 \varphi\left(R_{1}, \cdots, R_{n}\right)} .
\end{aligned}
$$

This implies that for each $t \in \mathbb{R}^{n}$

$$
c_{\alpha}(\varphi) \geqslant \frac{\pi^{n}}{\tilde{\alpha}_{1} \cdots \tilde{\alpha}_{n}} e^{\langle 2 \tilde{\alpha}, t\rangle-2 \varphi[e](t)} .
$$

Therefore,

$$
c_{\alpha}(\varphi) \geqslant \frac{\pi^{n}}{\tilde{\alpha}_{1} \cdots \tilde{\alpha}_{n}} e^{2(\varphi[e])^{*}(\tilde{\alpha})} .
$$

Employing now Proposition 2, we obtain easily the second statement of the lemma.

Lemma 3. Assume that an entire in $\mathbb{C}^{n}$ function satisfies $f(z)=\sum_{|\alpha| \geqslant 0} a_{\alpha} z^{\alpha} \in F_{\varphi}^{2}$. Then

$$
\sum_{|\alpha| \geqslant 0}\left|a_{\alpha}\right|^{2} c_{\alpha}(\varphi)<\infty \quad \text { and } \quad\|f\|_{\varphi}^{2}=\sum_{|\alpha| \geqslant 0}\left|a_{\alpha}\right|^{2} c_{\alpha}(\varphi)
$$

And vice versa, let the sequence $\left(a_{\alpha}\right)_{|\alpha| \geqslant 0}$ of complex number $a_{\alpha}$ is such that the series $\sum_{|\alpha| \geqslant 0}\left|a_{\alpha}\right|^{2} c_{\alpha}(\varphi)$ converges. Then $f(z)=\sum_{|\alpha| \geqslant 0} a_{\alpha} z^{\alpha} \in H\left(\mathbb{C}^{n}\right)$. At that, $f \in F_{\varphi}^{2}$.

Proof. Let

$$
f(z)=\sum_{|\alpha| \geqslant 0} a_{\alpha} z^{\alpha}
$$

be an entire function in $\mathbb{C}^{n}$ in the class $F_{\varphi}^{2}$. Then

$$
\begin{aligned}
\|f\|_{\varphi}^{2} & =\int_{\mathbb{C}^{n}}|f(z)|^{2} e^{-2 \varphi(\text { abs } z)} d \lambda(z)=\int_{\mathbb{C}^{n}} \sum_{|\alpha| \geqslant 0} a_{\alpha} z^{\alpha} \sum_{|\beta| \geqslant 0} \bar{a}_{\beta} \bar{z}^{\beta} e^{-2 \varphi(\text { abs } z)} d \mu_{n}(z) \\
& =\sum_{|\alpha| \geqslant 0}\left|a_{\alpha}\right|^{2} \int_{\mathbb{C}^{n}}\left|z_{1}\right|^{2 \alpha_{1}} \cdots\left|z_{n}\right|^{2 \alpha_{n}} e^{-2 \varphi(\operatorname{abs} z)} d \mu_{n}(z)=\sum_{|\alpha| \geqslant 0}\left|a_{\alpha}\right|^{2} c_{\alpha}(\varphi) .
\end{aligned}
$$

Vice versa, the convergence of the series $\sum_{|\alpha| \geqslant 0}\left|a_{\alpha}\right|^{2} c_{\alpha}(\varphi)$ and Lemma 2 implies that for each $\varepsilon>0$ there exists a constant $c_{\varepsilon}>0$ such that $\left|a_{\alpha}\right| \leqslant c_{\varepsilon} \varepsilon^{|\alpha|}$ for each $\alpha \in \mathbb{Z}_{+}^{n}$. This means that $f(z)=\sum_{|\alpha| \geqslant 0} a_{\alpha} z^{\alpha}$ is an entire function in $\mathbb{C}^{n}$. It is easy to see that $f \in F_{\varphi}^{2}$.

Lemma 4. Let $\varphi \in \mathcal{V}\left(\mathbb{R}^{n}\right)$. Then

$$
(2 \pi)^{n} e^{-1} V\left(D_{\tilde{\alpha}}^{\varphi[e]}\left(\frac{1}{2}\right)\right) e^{2(\varphi[e])^{*}(\tilde{\alpha})} \leqslant c_{\alpha}(\varphi) \leqslant(2 \pi)^{n}(1+n !) V\left(D_{\tilde{\alpha}}^{\varphi[e]}\left(\frac{1}{2}\right)\right) e^{2(\varphi[e])^{*}(\tilde{\alpha})}
$$

for each $\alpha \in \mathbb{Z}_{+}^{n}$. 
Proof. Let $\alpha=\left(\alpha_{1}, \ldots, \alpha_{n}\right) \in \mathbb{Z}_{+}^{n}$. Then

$$
\begin{aligned}
c_{\alpha}(\varphi) & =(2 \pi)^{n} \int_{0}^{\infty} \cdots \int_{0}^{\infty} r_{1}^{2 \alpha_{1}+1} \cdots r_{n}^{2 \alpha_{n}+1} e^{-2 \varphi\left(r_{1}, \cdots, r_{n}\right)} d r_{1} \cdots d r_{n} \\
& =(2 \pi)^{n} \int_{-\infty}^{\infty} \cdots \int_{-\infty}^{\infty} e^{\left(2 \alpha_{1}+2\right) t_{1}+\cdots+\left(2 \alpha_{n}+2\right) t_{n}-2 \varphi[e]\left(t_{1}, \ldots, t_{n}\right)} d t_{1} \cdots d t_{n} .
\end{aligned}
$$

That is,

By Theorem B we have

$$
c_{\alpha}(\varphi)=(2 \pi)^{n} \int_{\mathbb{R}^{n}} e^{\langle 2 \tilde{\alpha}, t\rangle-2 \varphi[e](t)} d t .
$$

$$
(2 \pi)^{n} e^{-1} V\left(D_{2 \tilde{\alpha}}^{2 \varphi[e]}\right) e^{2(\varphi[e])^{*}(\tilde{\alpha})} \leqslant c_{\alpha}(\varphi) \leqslant(2 \pi)^{n}(1+n !) V\left(D_{2 \tilde{\alpha}}^{2 \varphi[e]}\right) e^{2(\varphi[e])^{*}(\tilde{\alpha})}
$$

Since $D_{2 \tilde{\alpha}}^{2 \varphi[e]}=D_{\tilde{\alpha}}^{\varphi[e]}\left(\frac{1}{2}\right)$, by the previous inequality this completes the proof.

3.2. Proof of Theorem. Let us prove that the mapping $\mathcal{L}$ acts from $\left(F_{\varphi}^{2}\right)^{*}$ into $F_{\varphi^{*}}^{2}$. Let $S \in\left(F_{\varphi}^{2}\right)^{*}$. Then there exists a function $g_{S} \in F_{\varphi}^{2}$ such that $S(f)=\left(f, g_{S}\right)_{\varphi}$, that is,

$$
S(f)=\int_{\mathbb{C}^{n}} f(z) \overline{g_{S}(z)} e^{-2 \varphi(\operatorname{abs} z)} d \mu_{n}(z), \quad f \in F_{\varphi}^{2} .
$$

At that, $\|S\|=\left\|g_{S}\right\|_{\varphi}$. If $g_{S}(z)=\sum_{|\alpha| \geqslant 0} b_{\alpha} z^{\alpha}$, then $\hat{S}(\lambda)=\sum_{|\alpha| \geqslant 0} \frac{c_{\alpha}(\varphi) \overline{b_{\alpha}}}{\alpha !} \lambda^{\alpha}, \lambda \in \mathbb{C}^{n}$. Therefore,

$$
\|\hat{S}\|_{\varphi^{*}}^{2}=\sum_{|\alpha| \geqslant 0}\left(\frac{c_{\alpha}(\varphi)\left|b_{\alpha}\right|}{\alpha !}\right)^{2} c_{\alpha}\left(\varphi^{*}\right)
$$

By Lemma 3,

$$
\begin{aligned}
& \left.c_{\alpha}(\varphi) \leqslant(2 \pi)^{n}(1+n !) V\left(D_{\tilde{\alpha}}^{\varphi[e]}\left(\frac{1}{2}\right)\right)\right) e^{2(\varphi[e])^{*}(\tilde{\alpha})} \\
& c_{\alpha}\left(\varphi^{*}\right) \leqslant(2 \pi)^{n}(1+n !) V\left(D_{\tilde{\alpha}}^{\varphi^{*}[e]}\left(\frac{1}{2}\right)\right) e^{2\left(\varphi^{*}[e]\right)^{*}(\tilde{\alpha})}
\end{aligned}
$$

for each $\alpha \in \mathbb{Z}_{+}^{n}$.

Therefore,

$$
c_{\alpha}(\varphi) c_{\alpha}\left(\varphi^{*}\right) \leqslant(2 \pi)^{2 n}(1+n !)^{2} V\left(D_{\tilde{\alpha}}^{\varphi[e]}\left(\frac{1}{2}\right)\right) V\left(D_{\tilde{\alpha}}^{\varphi^{*}[e]}\left(\frac{1}{2}\right)\right) e^{2(\varphi[e])^{*}(\tilde{\alpha})+2\left(\varphi^{*}[e]\right)^{*}(\tilde{\alpha})}
$$

for each $\alpha \in \mathbb{Z}_{+}^{n}$.

According Proposition 6 , for each $\alpha=\left(\alpha_{1}, \ldots, \alpha_{n}\right) \in \mathbb{Z}_{+}^{n}$ we have

$$
(\varphi[e])^{*}(\tilde{\alpha})+\left(\varphi^{*}[e]\right)^{*}(\tilde{\alpha})=\sum_{j=1}^{n}\left(\left(\alpha_{j}+1\right) \ln \left(\alpha_{j}+1\right)-\left(\alpha_{j}+1\right)\right) .
$$

Since by the Stirling's formula [10], for each $m \in \mathbb{Z}_{+}$we have

$$
(m+1) \ln (m+1)-(m+1)=\ln \Gamma(m+1)-\ln \sqrt{2 \pi}+\frac{1}{2} \ln (m+1)-\frac{\theta}{12(m+1)},
$$

where $\theta \in(0,1)$ depends on $m$, then

$$
(\varphi[e])^{*}(\tilde{\alpha})+\left(\varphi^{*}[e]\right)^{*}(\tilde{\alpha})=-n \ln \sqrt{2 \pi}+\sum_{j=1}^{n}\left(\ln \Gamma\left(\alpha_{j}+1\right)+\frac{1}{2} \ln \left(\alpha_{j}+1\right)-\frac{\theta_{j}}{12\left(\alpha_{j}+1\right)}\right)
$$


where $\theta_{j} \in(0,1)$ depends on $\alpha_{j}$. Then

$$
\frac{e^{2\left((\varphi[e])^{*}(\tilde{\alpha})+\left(\varphi^{*}[e]\right)^{*}(\tilde{\alpha})\right)}}{\alpha !^{2}}=\frac{1}{(2 \pi)^{n}} \prod_{j=1}^{n}\left(\alpha_{j}+1\right) e^{-\frac{\theta_{j}}{6\left(\alpha_{j}+1\right)}} .
$$

Thus,

$$
\frac{c_{\alpha}(\varphi) c_{\alpha}\left(\varphi^{*}\right)}{\alpha !^{2}} \leqslant(2 \pi)^{n}(1+n !)^{2} V\left(D_{\tilde{\alpha}}^{\varphi[e]}\left(\frac{1}{2}\right)\right) V\left(D_{\tilde{\alpha}}^{\varphi^{*}[e]}\left(\frac{1}{2}\right)\right) \prod_{j=1}^{n} \tilde{\alpha}_{j} .
$$

Employing the condition for $\varphi$, we obtain that

$$
\frac{c_{\alpha}(\varphi) c_{\alpha}\left(\varphi^{*}\right)}{\alpha !^{2}} \leqslant(2 \pi)^{n}(1+n !)^{2} K
$$

for each $\alpha \in \mathbb{Z}_{+}^{n}$. Letting $M_{1}=(2 \pi)^{n}(1+n !)^{2} K$, by (1) we obtain

$$
\|\hat{S}\|_{\varphi^{*}}^{2} \leqslant M_{1} \sum_{|\alpha| \geqslant 0} c_{\alpha}(\varphi)\left|b_{\alpha}\right|^{2}=M_{1}\left\|g_{S}\right\|_{\varphi}^{2}=M_{1}\|S\|^{2} .
$$

Hence, $\hat{S} \in F_{\varphi^{*}}^{2}$. Moreover, the latter estimate implies that the linear mapping $\mathcal{L}$ acts continuously from $\left(F_{\varphi}^{2}\right)^{*}$ into $F_{\varphi^{*}}^{2}$.

We observe that the mapping $\mathcal{L}$ is injective from $\left(F_{\varphi}^{2}\right)^{*}$ into $F_{\varphi^{*}}^{2}$ since by Lemma 1 the system $\{\exp \langle\lambda, z\rangle\}_{\lambda \in \mathbb{C}^{n}}$ is complete in $F_{\varphi}^{2}$.

Let us show that the mapping $\mathcal{L}$ acts from $\left(F_{\varphi}^{2}\right)^{*}$ onto $F_{\varphi^{*}}^{2}$. Assume that $G \in F_{\varphi^{*}}^{2}$. Employing the representation of an entire function $G$ by the Taylor series

$$
G(\lambda)=\sum_{|\alpha| \geqslant 0} d_{\alpha} \lambda^{\alpha}, \quad \lambda \in \mathbb{C}^{n}
$$

we get

$$
\|G\|_{\varphi^{*}}^{2}=\sum_{|\alpha| \geqslant 0}\left|d_{\alpha}\right|^{2} c_{\alpha}\left(\varphi^{*}\right)
$$

For each $\alpha \in \mathbb{Z}_{+}^{n}$ we define the numbers $g_{\alpha}=\frac{\overline{d_{\alpha}} \alpha \text { ! }}{c_{\alpha}(\varphi)}$ and consider the convergence of the series $\sum_{|\alpha| \geqslant 0}\left|g_{\alpha}\right|^{2} c_{\alpha}(\varphi)$. We have

$$
\sum_{|\alpha| \geqslant 0}\left|g_{\alpha}\right|^{2} c_{\alpha}(\varphi)=\sum_{|\alpha| \geqslant 0}\left|\frac{\overline{d_{\alpha}} \alpha !}{c_{\alpha}(\varphi)}\right|^{2} c_{\alpha}(\varphi)=\sum_{|\alpha| \geqslant 0} \frac{\alpha !^{2}}{c_{\alpha}(\varphi) c_{\alpha}\left(\varphi^{*}\right)}\left|d_{\alpha}\right|^{2} c_{\alpha}\left(\varphi^{*}\right) .
$$

By Lemma 4,

$$
c_{\alpha}(\varphi) \geqslant e^{-1} V\left(D_{\tilde{\alpha}}^{\varphi[e]}\left(\frac{1}{2}\right)\right) e^{2(\varphi[e])^{*}(\tilde{\alpha})}, \quad c_{\alpha}\left(\varphi^{*}\right) \geqslant e^{-1} V\left(D_{\tilde{\alpha}}^{\varphi^{*}[e]}\left(\frac{1}{2}\right)\right) e^{2\left(\varphi^{*}[e]\right)^{*}(\tilde{\alpha})}
$$

for each $\alpha \in \mathbb{Z}_{+}^{n}$. Therefore,

$$
c_{\alpha}(\varphi) c_{\alpha}\left(\varphi^{*}\right) \geqslant e^{-2} V\left(D_{\tilde{\alpha}}^{\varphi[e]}\left(\frac{1}{2}\right)\right) V\left(D_{\tilde{\alpha}}^{\varphi^{*}[e]}\left(\frac{1}{2}\right)\right) e^{2\left((\varphi[e])^{*}(\tilde{\alpha})+\left(\varphi^{*}[e]\right)^{*}(\tilde{\alpha})\right)}
$$

for each $\alpha \in \mathbb{Z}_{+}^{n}$. By identity (2) this implies

$$
\frac{\alpha !^{2}}{c_{\alpha}(\varphi) c_{\alpha}\left(\varphi^{*}\right)} \leqslant \frac{e^{2}(2 e \pi)^{n}}{V\left(D_{\tilde{\alpha}}^{\varphi[e]}\left(\frac{1}{2}\right)\right) V\left(D_{\tilde{\alpha}}^{\varphi^{*}[e]}\left(\frac{1}{2}\right)\right) \prod_{j=1}^{n}\left(\alpha_{j}+1\right)}
$$

for each $\alpha=\left(\alpha_{1}, \ldots, \alpha_{n}\right) \in \mathbb{Z}_{+}^{n}$. 
Employing the condition for $\varphi$, we obtain that $\frac{\alpha !^{2}}{c_{\alpha}(\varphi) c_{\alpha}\left(\varphi^{*}\right)} \leqslant K e^{2}(2 e \pi)^{n}, \forall \alpha \in \mathbb{Z}_{+}^{n}$. Therefore, for the considered series we have

$$
\sum_{|\alpha| \geqslant 0}\left|g_{\alpha}\right|^{2} c_{\alpha}(\varphi) \leqslant K e^{2}(2 e \pi)^{n} \sum_{|\alpha| \geqslant 0}\left|d_{\alpha}\right|^{2} c_{\alpha}\left(\varphi^{*}\right)=K e^{2}(2 e \pi)^{n}\|G\|_{\varphi^{*}}^{2} .
$$

Thus, the series $\sum_{|\alpha| \geqslant 0}\left|g_{\alpha}\right|^{2} c_{\alpha}(\varphi)$ converges. But by Lemma 3 the function

$$
g(\lambda)=\sum_{|\alpha| \geqslant 0} g_{\alpha} \lambda^{\alpha}, \quad \lambda \in \mathbb{C}^{n}
$$

is entire and by (3), $g$ belongs to $F_{\varphi}^{2}$ and

$$
\|g\|_{\varphi}^{2} \leqslant K e^{2}(2 e \pi)^{n}\|G\|_{\varphi^{*}}^{2}
$$

We define a functional $S$ on $F_{\varphi}^{2}$ by the formula

$$
S(f)=\int_{\mathbb{C}^{n}} f(z) \overline{g(z)} e^{-2 \varphi(\operatorname{abs} z)} d \mu_{n}(z), \quad f \in F_{\varphi}^{2} .
$$

It is clear that $S$ is a linear continuous functional on $F_{\varphi}^{2}$. At that, $\hat{S}=G$. Since $\|S\|=\|g\|_{\varphi}$, estimate (4) shows that the inverse mapping $\mathcal{L}^{-1}$ is continuous. Thus, $\mathcal{L}$ makes an isomorphism between the spaces $\left(F_{\varphi}^{2}\right)^{*}$ and $F_{\varphi^{*}}^{2}$. The proof is complete.

\section{BIBLIOGRAPHY}

1. I.Kh. Musin On a space of entire functions rapidly decreasing on $R^{n}$ and its Fourier transform // Concr. Operat. 2:1, 120-138 (2015).

2. D. Azagra. Global and fine approximation of convex functions // Proc. London Math. Soc. 107:4, 799-824 (2013).

3. D. Azagra Global approximation of convex functions // Preprint: arXiv:1112.1042v7.

4. B.A. Taylor. On weighted polynomial approximation of entire functions // Pacific J. Math. 36:2, 523-539 (1971).

5. V.V. Napalkov, S.V. Popënov. On the Laplace transform of functionals in the Bergman weight space of entire functions in $\mathbb{C}^{n} / /$ Dokl. Akad. Nauk. 352:5, 595-597. [Dokl. Math. 55:1, 110-112 (1997).]

6. S.V. Popenov. On Laplace transform of functionals in some weighted Bergman spaces in $\mathbb{C}^{n} / /$ Proc. Int. Conf. "Complex analysis, differential equations, numerical methods and applications". Ufa. 2, 125-132 (1996). (in Russian).

7. R.S. Yulmukhametov. Asymptotics of multi-dimensional Laplace integral // in "Studies in approximation theory", Ufa. 132-137 (1989). (in Russian).

8. V.V. Napalkov, R.A. Bashmakov, R.S.Yulmukhametov. Asymptotic behavior of Laplace integrals and geometric characteristics of convex functions // Dokl. Akad. Nauk. 413:1, 20-22 (2007). [Dokl. Math. 75:2, 190-192 (2007).]

9. R.A. Bashmakov, K.P. Isaev, R.S. Yulmukhametov. On geometric characteristics of convex function and Laplace integrals // Ufimskij Matem. Zhurn. 2:1, 3-16 (2010). (in Russian).

10. G.M. Fikhtengolts. Course of differential and integral calculus. V. II. Nauka, Moscow (1970). (in Russian).

Il'dar Khamitovich Musin

Institute of Mathematics, Ufa Scientific Center, RAS,

Chernyshevsky str. 112,

450008, Ufa, Russia

E-mail: musin_ildar@mail.ru 